\title{
Multi-harmonic LQ Control of Single-phase Converter with LCL filter
}

\author{
Štěpán Bláha ${ }^{1}$, Tomáš Komrska ${ }^{2}$, Václav Šmídl ${ }^{3}$, Vendula Glasbergerová ${ }^{4}$, Zdeněk Peroutka $^{5}$ \\ Regional Innovation Centre for Electrical Engineering (RICE), Faculty of Electrical Engineering, \\ University of West Bohemia, Univerzitni 26, 30614 Pilsen, Czech Republic \\ E-mail: ${ }^{1}$ blahast@kev.zcu.cz, ${ }^{2}$ komrska@ rice.zcu.cz, ${ }^{3}$ vsmidl@ ${ }_{\text {rice.zcu.cz, }}{ }^{4}$ vendulam@kev.zcu.cz, \\ 5 peroutka@ieee.org
}

\begin{abstract}
Control of single-phase converters connected to the grid via an LCL filter is a demanding issue due to risk of oscillations. A linear-quadratic (LQ) controller enabling besides standard pure sine control also a multiharmonic current reference is proposed in the paper. Such a design enables to control single-phase converters generating harmonic currents, e.g. shunt active filters, or converters operating under distorted voltage conditions. In order to reduce number of sensors, the proposed LQ control has been completed by a state estimator based on the Kalman filter.
\end{abstract}

Index Terms-control, multi-harmonic, single-phase, LCL filter, linear-quadratic

\section{INTRODUCTION}

Due to the changes in the trend of electricity distribution and production, there is an increase in number of grid-connected converters. The disturbances caused by converters leads to necessity of connection LCL filters between the converter and grid. Usage of these filters has advantage in better attenuation of switching harmonics, which leads to minimization of the current ripple followed by cost reduction of the filter components . However it brings problems with oscillations at resonance frequency of LCL filter.

The most of the applications of grid-connected converters with LCL filter are in the three-phase power systems [1],[2],[3],[4], but only a few of these applications are related to single-phase systems [5]. Control techniques designed for 3-phase systems usually utilize $d q$ decomposition, which is problematic in the singlephase systems (virtual $q$ component). From the control point of view there are standard solutions like the proportional resonant current control [6] or less standard solutions like with usage of neural network [7].

Besides the active rectifiers and inverters are employed like shunt active power filters [8] or current injectors (impedance estimation), where not only generation of the sinusoidal current with grid frequency is required, but where the reference current consists of multiple harmonic elements [9].

The control strategy proposed in this article is based on linear-quadratic control. It is capable of operation under distorted voltage conditions and to generate multi-harmonic current reference, so this control strategy is suitable for previously mentioned applications.

The standard control of grid-connected converters with LCL filters requires multiple current and voltage sensors on both grid and converted sides. In case of proposed control strategy the number of sensors is minimized by usage of state observer based on Kalman filter.

\section{SySTEM DESCRIPTION}

The single-phase grid-connected converter with LCL filter is shown in Fig. 1. From DC side the converter is powered by voltage $U_{d c}$. The objective of the control is variable $i_{2}$ - grid current. The vector of the state variables for this system consists of 5 elements currents $i_{1}, i_{2}$, capacitor voltage $u_{c}$, grid voltage $u_{g}$ and its derivative.

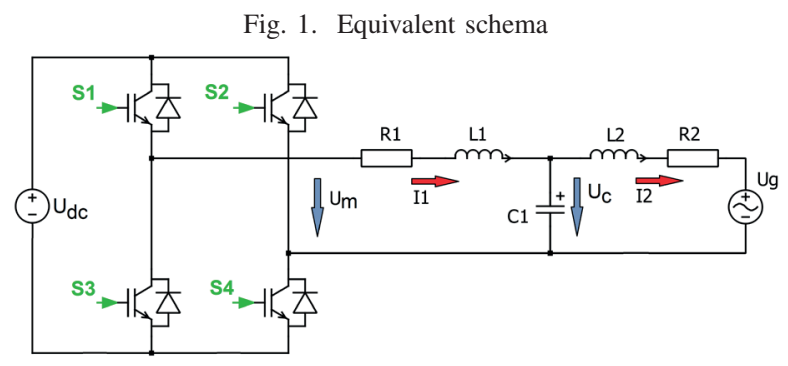

For prediction of the system trajectory it is necessary to consider grid voltage as a state variable. For this purpose the grid voltage is modeled as an undamped oscillator, as discussed below.

The equation (1) gives a general description of the system in continuous time form. It defines a general relation between state variables, their derivatives and input variable, which is represented by voltage source $u_{m}$. The parameters $A$ and $B$ (2) represent state space matrices of the given system (as a set of 5 equations).

$$
\frac{d x(t)}{d t}=A x(t)+B u(t)
$$

with

$$
A=\left[\begin{array}{ccccc}
\frac{-R_{1}}{L_{1}} & 0 & \frac{-1}{L_{1}} & 0 & 0 \\
0 & \frac{-R_{2}}{L_{2}} & \frac{1}{L_{2}} & \frac{-1}{L_{2}} & 0 \\
\frac{1}{C} & \frac{-1}{C} & 0 & 0 & 0 \\
0 & 0 & 0 & 0 & 1 \\
0 & 0 & 0 & -\omega^{2} & 0
\end{array}\right], B=\left[\begin{array}{c}
\frac{1}{L_{1}} \\
0 \\
0 \\
0 \\
0
\end{array}\right]
$$

The relation between state variables of LCL filter $\left(i_{1}, i_{2}, u_{c}\right)$ and input voltage source $u_{m}$ is defined by 
the first 3 equations of the set (1). Grid voltage $u_{g}$ is modeled as a harmonic oscillator (3), where $\omega$ represents frequency of the oscillations (in this case $\omega=2 \pi 50)$.

$$
\frac{d^{2} u_{g}}{d t}=-\omega^{2} u_{g} \Rightarrow \frac{d}{d t}\left[\begin{array}{l}
u_{g} \\
u_{g}^{\prime}
\end{array}\right]=\left[\begin{array}{cc}
0 & 1 \\
-\omega^{2} & 0
\end{array}\right]\left[\begin{array}{l}
u_{g} \\
u_{g}^{\prime}
\end{array}\right]
$$

The continuous time model (1) is converted to discrete time model

$$
x_{k+1}=A_{d} x_{k}+B_{d} u_{k}
$$

using the standard discretization procedure

$$
\begin{aligned}
& A_{d}=e^{A \Delta t} \\
& B_{d}=A^{-1}\left(A_{d}-I\right) B
\end{aligned}
$$

\section{OBSERVATION MODELS}

Since the state variable of model (1) is not fully measured (the derivative of the grid voltage $u_{g}^{\prime}$ needs to be calculated), the observation equation is defined as:

$$
y_{k}=C x_{k}
$$

where $C$ is the observation matrix. Only two scenarios will be considered where all state elements are observed $y_{k}^{(1)}=\left[i_{1, k}, i_{2, k}, u_{c, k}, u_{g, k}\right]$ and where only 2 state elements are observed, namely $y_{k}^{(2)}=\left[i_{2, k}, u_{g, k}\right]$. This leads to observation matrices:

$$
\begin{aligned}
& C^{(1)}=\left[\begin{array}{lllll}
1 & 0 & 0 & 0 & 0 \\
0 & 1 & 0 & 0 & 0 \\
0 & 0 & 1 & 0 & 0 \\
0 & 0 & 0 & 1 & 0
\end{array}\right] \\
& C^{(2)}=\left[\begin{array}{lllll}
0 & 1 & 0 & 0 & 0 \\
0 & 0 & 0 & 1 & 0
\end{array}\right]
\end{aligned}
$$

Each of these observation matrices implies own design of the Kalman filter

$$
\hat{x}_{k}=A_{d} \hat{x}_{k-1}+B_{d} u_{k}+K^{(i)}\left(y_{k}-C^{(i)} \hat{x}_{k-1}\right) .
$$

Since the system is linear the Kalman filter [10] converges to a steady state solution of the Kalman gain $K^{(i)}$ for each $C^{(i)}$ and appropriate covariance matrices of the residues.

\section{LQ CONTROL STRATEGY}

Since the system is linear, we can use the linearquadratic control design. In case of tracking of multiharmonic reference it comes to minize quadratic cost function (10), where $i_{2, \kappa}^{*}$ represents the sum of all required harmonics.

$$
\begin{gathered}
g=\sum_{\kappa=k+1}^{\infty}\left(i_{2, \kappa}-i_{2, \kappa}^{*}\right)^{2}, \\
i_{2, \kappa}^{*}=\sum_{n}^{N} I_{2, n}^{*} \sin \left(\omega_{n} \kappa \Delta t+\varphi_{2, n}\right)
\end{gathered}
$$

Note that the sinusoidal reference can be again modeled by a single undamped oscillator.

$$
\frac{d}{d t}\left[\begin{array}{c}
i_{2}^{*} \\
d i_{2}^{*}
\end{array}\right]=\left[\begin{array}{cc}
0 & 1 \\
-\omega^{2} & 0
\end{array}\right]\left[\begin{array}{c}
i_{2}^{*} \\
d i_{2}^{*}
\end{array}\right] .
$$

In case of tracking the multi-harmonic reference the series of undamped oscillators is needed (13). The resonance frequency of each oscillator refers to required harmonic component.

$$
\frac{d}{d t} \phi_{n}=A_{\phi_{n}} \phi_{n}
$$

where

$$
\phi_{n}=\left[\begin{array}{c}
i_{2(n)}^{*} \\
d i_{2(n)}^{*}
\end{array}\right], A_{\phi_{n}}=\left[\begin{array}{cc}
0 & 1 \\
-\omega_{(n)}^{2} & 0
\end{array}\right]
$$

The tracking problem then can be written in standard LQ formulation with an augmented state, $\tilde{x}_{k}=$ $\left[i_{1, k}, i_{2, k}, u_{c, k}, u_{g, k}, u_{g, k}^{\prime}, \phi_{1} \ldots \phi_{N}\right]$ with model:

$\frac{d}{d t}\left[\begin{array}{c}x \\ \phi_{1} \\ \vdots \\ \phi_{N}\end{array}\right]=\left[\begin{array}{cccc}A & 0 & \ldots & 0 \\ 0 & A_{\phi_{1}} & \ldots & 0 \\ \vdots & \vdots & \ddots & 0 \\ 0 & 0 & 0 & A_{\phi_{N}}\end{array}\right]\left[\begin{array}{c}x \\ \phi_{1} \\ \vdots \\ \phi_{N}\end{array}\right]+\left[\begin{array}{c}B \\ 0 \\ \vdots \\ 0\end{array}\right]_{(14)}$

The transformation of the model into the discrete time using standard formula is needed. The extended matrices will be denoted by $\tilde{A}_{d}$ and $\tilde{B}_{d}(5)$.

The control objective (10) can be written as

$$
\begin{aligned}
Z \tilde{x}_{k} & =i_{2, k}-i_{2, k}^{*} \\
Z & =\left[\begin{array}{llllllllll}
0 & 1 & 0 & 0 & 0 & -1 & 0 & \ldots & -1 & 0
\end{array}\right]
\end{aligned}
$$

which is in standard quadratic form

$$
g=\sum_{\kappa=k}^{\infty} \tilde{x}_{\kappa}^{T} Q \tilde{x}_{\kappa},
$$

with

$$
Q=Z^{T} Z
$$

Note that the matrix $Q$ is not positive definite which is a common requirement of LQ design approaches. Therefore, a routine suitable for semi-definite matrices has to be used (e.g. those based on QR decomposition). The resulting controller is:

$$
u_{k}^{L Q}=-L \tilde{x}_{k} .
$$

Using the principle of separation, the state $\tilde{x}_{k}$ is replaced by its estimate from the Kalman filter (9).

\section{Simulation}

The simulation process is running in the environment of Matlab/Simulink. It consists of several parts as it is shown in the block diagram (Fig. 2). Fundamental block of the mathematical model is singlephase converter with LCL filter assembled in the Plecs environment. This block is used as a reference and its outputs (state variables) are considered as a measurements for the simulation. These outputs are connected to the observation/estimation block, which is based on Kalman filter.

In the first phase (Fig. 3) only single sinusoidal reference is required. The Kalman filter is used only for the estimation of the derivative of grid voltage $u_{g}$ with usage of measurement of all remaining state variables. In the next phase (Fig. 4) the reference is the same but the Kalman filter is using only the measurement of the 
output current $i_{2}$ and grid voltage $u_{g}$. All remaining state variables are estimated and used for LQ control.

The block of LQ control stands for the multiplication of optimal linear gains $L$ (calculated at the begin of the simulation) and the output vector of the state variables from the Kalman filter as it is shown in (18). The output of LQ control is considered as a reference input signal for the block of PWM generator. This block is directly connected to the converter in the Plecs environment.

Fig. 2. Block diagram

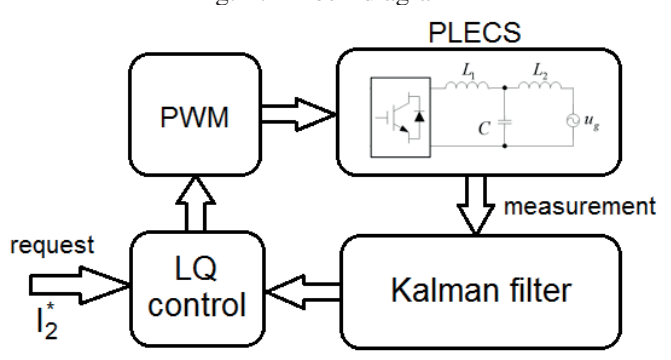

Fig. 3 shows the results of the simulation for the main state variables of the system. This simulation is based on the observation vector $y_{k}^{(1)}=$ $\left[i_{1, k}, i_{2, k}, u_{c, k}, u_{g, k}\right]$. The proposed LQ controller is designed to control the output current of LCL filter $i_{2}$ and in this case current is controlled to 2 different levels with change in phase of the current. To test this scenario the change in the amplitude of grid voltage $u_{g}$ is also applied.

Fig. 3. Simulation results: LQ control of the system with measurements of all state variables
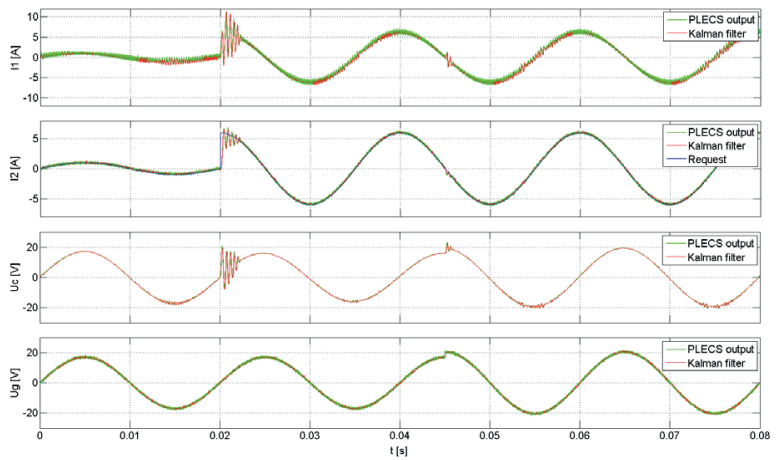

Fig. 4 shows the same state variables of the system as the previous figure. In this case the simulation is based on the observation vector $y_{k}^{(2)}=\left[i_{2, k}, u_{g, k}\right]$. The output current $i_{2}$ is driven to the same levels, phase and grid voltage changes as in the previous scenario.

In the third phase (shown in Fig. 5) the multiharmonic reference is required. The reference multiharmonic signal consists of 3 elementary harmonics created by multiplication of base frequency $(50 \mathrm{~Hz}, 150 \mathrm{~Hz}, 250 \mathrm{~Hz})$. This simulation is based on the observation vector $y_{k}^{(1)}=\left[i_{1, k}, i_{2, k}, u_{c, k}, u_{g, k}\right]$. The output current $i_{2}$ is again controlled to 2 different levels and the change in the amplitude of grid voltage $u_{g}$ is also applied for comparison.
Fig. 4. Simulation results: LQ control of the system with measurements of $i_{2}$ and $u_{g}$

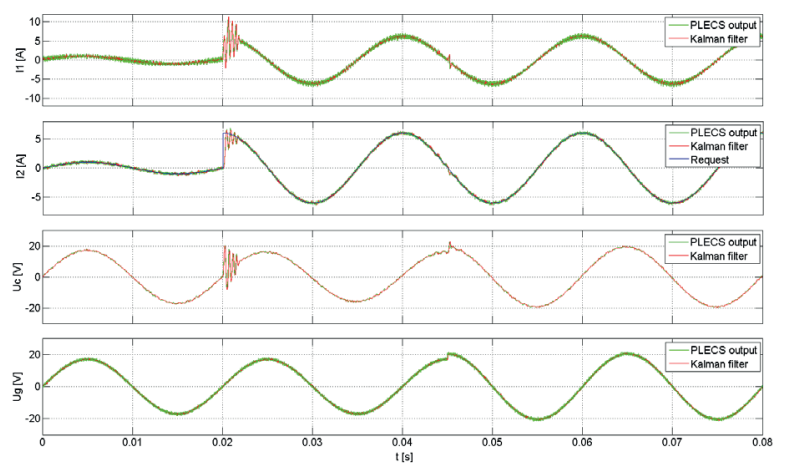

The last phase (Fig. 6) represents the same multiharmonic reference and control levels, but in this case the simulation is based on the observation vector $y_{k}^{(2)}=\left[i_{2, k}, u_{g, k}\right]$.

Fig. 5. Simulation results: LQ control of the system with multiharmonic reference (measurements of all state variables)

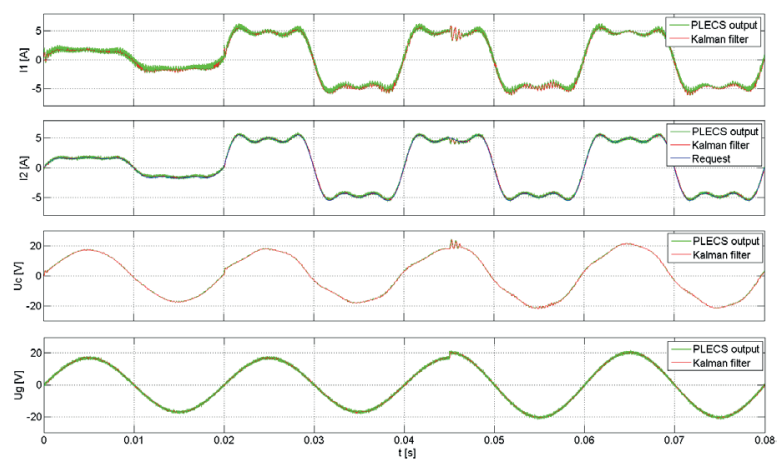

Fig. 6. Simulation results: LQ control of the system with multiharmonic reference (measurements of $i_{2}$ and $u_{g}$ )
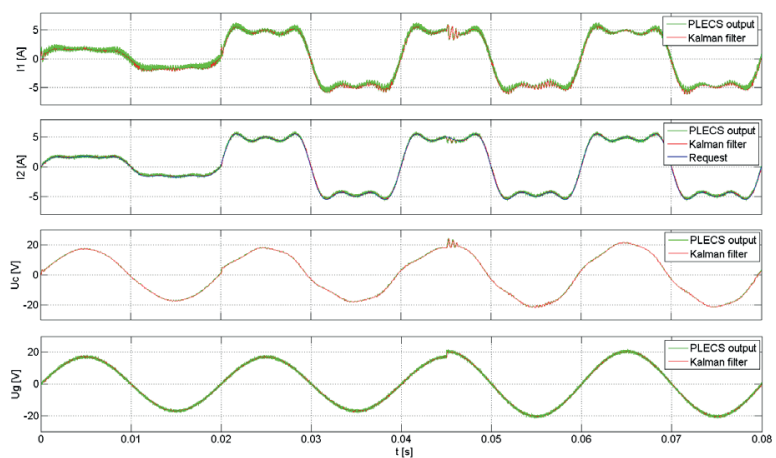

\section{CONCLUSION}

Since the single-phase grid-connected converter can be applied as active power filter or for current injection to estimate impedance, where multi-harmonic current reference is required, the proposed control has been designed with modeled oscillators in the state model.

Simulation results indicate that proposed control exhibit proper operation in the tracking of sinusoidal 
reference as well as in case of tracking the multiharmonic reference.

Performed simulation results verify correct operation of proposed control for both configurations, i.e. 1) all state variables are measured, 2) only grid voltage and current are measured; remaining state variables are estimated by the Kalman filter.

Simulation results indicate that proposed control exhibit proper operation in steady state as well as good behavior during transients (step change in demanded current amplitude and phase angle). The proposed control also acceptable response to step change in the amplitude of grid voltage.

\section{ACKNOWLEDGMENT}

This work was created under the project No. SGS2015-038.

\section{REFERENCES}

[1] J. R. Massing, M. Stefanello, H. A. Grundling, and H. Pinheiro, "Adaptive current control for grid-connected converters with lcl filter," IEEE Transactions on Industrial Electronics, vol. vol. 59, no. issue 12, pp. 4681-4693, 2012.

[2] J. Kukkola and M. Hinkkanen, "State observer for grid-voltage sensorless control of a grid-connected converter equipped with an 1cl filter," 2014 16th European Conference on Power Electronics and Applications, pp. 1-10, 2014.
[3] B.-G. Cho, S.-K. Sul, H. Yoo, and S.-M. Lee, "Lcl filter design and control for grid-connected pwm converter," 8th International Conference on Power Electronics - ECCE Asia, pp. 756-763, 2011.

[4] J. Kukkola and M. Hinkkanen, "Observer-based state-space current control for a three-phase grid-connected converter equipped with an lcl filter," 2013 IEEE Energy Conversion Congress and Exposition, pp. 1371-1378, 2013.

[5] G. Escobar, S. Pettersson, and C. Ho, "Control of single-phase inverter connected to the grid through an lcl filter," IECON 2012 - 38th Annual Conference on IEEE Industrial Electronics Society, pp. 3406-3411, 2012.

[6] G. Shen, X. Zhu, M. Chen, and D. Xu, "A new current feedback pr control strategy for grid-connected vsi with an lcl filter," 2009 Twenty-Fourth Annual IEEE Applied Power Electronics Conference and Exposition, pp. 1564-1569, 2009.

[7] X. Fu and S. Li, "Control of single-phase grid-connected converters with lcl filters using recurrent neural network and conventional control methods," IEEE Transactions on Power Electronics, pp. 1-1, 2015.

[8] H. Akagi, "Active harmonic filters," Proceedings of the IEEE, vol. vol. 93, no. issue 12, pp. 2128-2141, 2005.

[9] Z. Yang, H. Shi, X. Yue, L. Hou, and F. Zhuo, "Harmonic impedance measurement for an islanded microgrid using current injection."

[10] A. P. A. Mohinder S. Grewal, Angus P. Andrews. Mohinder $\mathrm{S}$. Grewal, Kalman filtering theory and practice using MAT$L A B$, 3rd ed. Hoboken, N.J: Wiley, 2008. 